\title{
Hem-o-lok clips are safe and efficacious for laparoscopic cholecystectomy: Preliminary results of a single center experience
}

\author{
Gaetano Poillucci, *; Francesca Frangella ${ }^{1}$; Piero Liberatore ${ }^{1}$; Renato De Angelis ${ }^{2}$ \\ ${ }^{1}$ Department of General and Specialized Surgery "Paride Stefanini", Policlinico Universitario Umberto I, Rome, Italy. \\ ${ }^{2}$ Department of General Surgery, San Giovanni Addolorata Hospital, Rome, Italy.
}

\section{*Corresponding Author: Gaetano Poillucci}

Department of General and Specialized Surgery

"Paride Stefanini", Policlinico Universitario Umberto

I, Rome, Italy, Viale del Policlinico, 155, 00161 Rome, Italy.

Tel: 0039-3408943471;

Email: gaetano.poillucci@gmail.com

Received: Aug 26, 2021

Accepted: Sep 22, 2021

Published: Sep 24, 2021

Archived: www.jjgastro.com

Copyright: (C) Poillucci G (2021).

\section{Abstract}

Introduction: The aim of this retrospective study was to compare the outcomes after laparoscopic cholecystectomy using titanium clips or Hem-o-lok polymeric clips to close cystic artery and cystic duct.

Methods: A total of 154 patients who underwent laparoscopic cholecystectomy for gallbladder disease from January 2019 to December 2019 at the Department of Surgery of the San Giovanni Addolorata Hospital in Rome (Italy) were studied retrospectively. In 100 patients, titanium clips were used, while Hem-o-lok clips were used in 54 patients. In the two groups, patients' demographic characteristics, laboratory tests and postoperative complications were analyzed.

Results: The mean length of hospital stay was shorter in the Hemo-lok group compared to the titanium clips group (2.0 SD0.7 vs. 3.6 SD5.5, $\mathrm{p}<0.05)$. The overall complication rates for the titanium clips and the Hem-o-lock clips groups were $9 \%$ and $0 \% 7$ days after surgery ( $p<0.05$ ), 10\% and $9.3 \% 30$ days after surgery, 13\% and $16.7 \% 180$ days after surgery. There were no statistically significant differences between the two groups in the typology of complications.

Conclusion: Based on our results, Hem-o-lock have better postoperative outcomes after laparoscopic cholecystectomy for gallbladder disease in terms of complication rate 7 days after surgery and in terms of mean length of hospital stay.

Keywords: laparoscopic cholecystectomy; Hem-O-Lok polymeric clips; titanium clips; biliary leakage; retrospective study.

Abbreviations: LC: Laparoscopic Cholecystectomy; TC: Titanium Clips; HC: Hem-o-lok Clips; STROBE: Strengthening the Reporting of Observational Studies in Epidemiology; ERCP: Endoscopic Retrograde CholangioPancreatography; WBC: White Blood Cell; CRP: C-Reactive Protein; PA: Phosphatase Alkaline; gGT: gamma Glutamyl Transferase; SD: Standard Deviation; FDA: Food and Drug Administration; MAUDE: MAnufacturer and User facility Device Experience. 
Citation: Poillucci G, Frangella F, Liberatore P, Angelis RD. Hem-o-lok clips are safe and efficacious for laparoscopic cholecystectomy: Preliminary results of a single center experience. Japanese J Gastroenterol Res. 2021; 1(4): 1020.

\section{Introduction}

Gallstone disease is one of the most common digestive diseases and affects $20 \%$ of the adult population in Europe [1]. The estimated prevalence of gallstone disease ranges between $5 \%$ and $22 \%$, depending on risk factors [2]. Approximately 20 to $40 \%$ of patients with asymptomatic cholelithiasis will develop symptoms during their lifetimes [3] and some patients also have severe complications [4]. Gallstone disease can be complicated by acute calculous cholecystitis $(0.3 \%-0.4 \%$ annually), acute biliary pancreatitis ( $0.04 \%-1.5 \%$ annually), common bile duct stones ( $0.1 \%-0.4 \%$ annually), and cholangitis $(0.3 \%-1.6 \%$ annually) [5]. Approximately $85 \%$ of patients with symptomatic gallstone disease have symptoms without signs of complications [6]. Patients usually report less specific abdominal symptoms such as dyspeptic symptoms or chronic abdominal pain. Currently, conservative treatment is recommended for patients with asymptomatic gallstones, while for those with symptomatic gallstone disease cholecystectomy is recommended. Cholecystectomy rates vary widely in Europe, with low annual rates in Norway and Denmark (62 - 68 per 1,00,000 inhabitants, respectively) and high rates in Belgium and Germany (189 - 246 per 100000 inhabitants, respectively) [1]. In the United States, 700000 cholecystectomies are performed yearly (214 per 100000 inhabitants) [2].

Laparoscopic Cholecystectomy (LC) is the gold standard for surgical treatment of gallstone disease: it has been shown that LC has many advantages as compared with the open approach technique, including less postoperative pain, faster recovery, shorter hospital stays, good cosmetic effect and lower percentage of surgical site infections [7].

There are several methods for ligating structures such as cystic arteries and cystic ducts during LC. Each technique has potential drawbacks. Application of endoloops requires dexterity and training; endoscopic staplers are more expensive instruments, and Titanium Clips (TC) can slip from their primary position [8]. Hem-o-lok Clips (HC) are nonabsorbable polymer clips that have a lock engagement feature, as well as teeth in the jaws that provide good security.

The aim of this retrospective study was to compare the outcomes after LC using TC or HC in terms of post-operative complications (rates of leakage from the cystic duct, intra-abdominal abscesses, intra-abdominal bleedings, wound infections, chronic abdominal pain, gastrointestinal symptoms and common bile duct stones).

\section{Materials and methods}

\section{Study population}

Informed consent was obtained from all individual participants included in the study, and all procedures were in accordance with the ethical standards of the institutional and/or national research committee and with the 1964 Helsinki declaration and its later amendments or comparable ethical standards.

The report of this study was prepared in accordance with the guidelines set by the Strengthening the Reporting of Observational Studies in Epidemiology (STROBE) statement for observational studies.

\section{Inclusion criteria}

- Signed informed consent for the treatment of personal and sensitive data;

- Patients older than 18 years of age;

- Diagnosis of gallbladder stones eventually confirmed by ultrasound and/or computed tomography scan in selected patients [9].

- LC.

\section{Exclusion criteria}

- Pregnant or lactating;

- Non-consenting patients;

- Open cholecystectomy or conversion during LC.

- Cholecystectomy performed during other abdominal surgical procedures.

\section{Surgical technique}

During a LC, two HCs were placed on the proximal part of the cystic duct, and only one was placed on the distal section; ligation of the cystic artery was also performed with application of a single clip. Instead, we applied two TCs for ligation of the cystic artery.

\section{Study endpoints}

Primary outcome: Treatment efficacy was defined as "complication-free treatment success based on 1-week, 1-month and 6-months follow-up" (success of the initial treatment with uncomplicated course: no post-operative complications, adverse events, or treatment failure occurring) in the TC group and in the $\mathrm{HC}$ group.

Secondary outcomes: Overall post-intervention complications: number and rate of leakage from the cystic duct, intraabdominal abscesses and intra-abdominal bleeding, wound infections, chronic abdominal pain, gastrointestinal symptons (dyspepsia and diarrhea), and common bile duct stones;

\section{Length of primary hospital stay.}

\section{Data collection and statistics}

All diagnosis and surgical operation codes were classified according to the International Classification of Diseases, 9th Edition, Clinical Modification (ICD-9-CM). Patients with a diagnosis of gallbladder stones with or without acute cholecystitis and without obstruction (574 and 574.00) who received LC during the period January 2019 - December 2019 at the Department of Surgery of the San Giovanni Addolorata Hospital in Rome (Italy) were identified and their data were collected, entered in a database (Excel 2010, Microsoft ${ }^{\circledR}$, Redmond, Washington, USA), and analyzed with STATA 15 (StataCorp. 2017. Stata Statistical Software: Release 15. College Station, TX, USA: StataCorp LLC).

Characteristics evaluated as categorical variables included gender, previous episodes of acute cholecystitis or acute pancreatitis, previous Endoscopic Retrograde CholangioPancreatography (ERCP), clips used, and comorbidity. 
Quantitative variables included age, White Blood Cell (WBC) count 24 hours after surgery, percentage of neutrophils on the first post-operative day, C-Reactive Protein (CRP) 24 hours after surgery, Phosphatase Alkaline (PA)/gamma Glutamyl Transferase (gGT) and total bilirubin 24 hours after surgery, length of hospital stay, complication rates after 7 days/30 days/180 days after LC.

Data were abstracted for each patient from the ward medical and outpatient clinic records.

Dichotomous variables are expressed as numbers and percentages, while continuous variables are mean and Standard Deviation (SD). Categorical variables were compared using Fisher's exact test and the continuous variable data were analyzed using Student's t-test. The level of statistical significance was established at $p<0.05$ (two-tailed model for unpaired data).

\section{Results}

During the period, 154 patients were treated with LC for gallbladder disease. As shown in (Table 1), 85 patients (55.2\%) were men, and $69(44.8 \%)$ were women. The mean age of the cohort was 56.7 SD14.8 years.

In this cohort, 80 patients (51.9\%) had had previous episodes of acute cholecystitis, 17 patients $(11 \%)$ previous episodes of acute pancreatitis and 11 patients (7.1\%) had been subjected to ERCP. We used TCs for the cystic artery and cystic duct in 100 patients (64.9\%) and HCs in 54 patients (35.1\%). A total of 46 patients $(29.9 \%)$ had comorbidities.

There was no statistically significant difference between the two groups in terms of gender, comorbidity or laboratory tests (except for CRP and gGT) as reported in (Table 2).

There were instead statistically significant differences between the two groups in mean age (54.0 SD13.7 in group TC vs. 61.7 SD15.5 in group HC, p-value $=0.0018)$, CRP serum level 24 hours after surgery (6.1 SD5.2 in group TC vs. 3.5 SD3.4 in group HC, $p$-value $=0.0012$ ), and gGT serum level 24 hours after surgery (46.7 SD44.9 in group TC group vs. 80.6 SD124.8 in group $\mathrm{HC}, \mathrm{p}$-value $=0.0157)$.

There was a statistically significant difference between the two groups in complication rate 7 days after LC ( $9 \%$ in group TC vs. $0 \%$ in group $\mathrm{HC}$, p-value $=0.0273$ ). No statistically significant differences between the two groups appeared 30 days and 180 days after surgery.

The overall complication rate was $5.8 \%$ (9 patients) 7 days after surgery, $9.7 \%$ (15 patients) 30 days after surgery, and $14.3 \%$ (22 patients) 180 days after surgery. The list of complications is shown in (Table 3). There were no statistically significant differences between the two groups.

Length of hospital stay was shorter in group $\mathrm{HC}$ compared to group TC (3.6 SD5.5 in group TC vs. 2.0 SD0.7 in group HC, $\mathrm{p}$-value $=0.0352$ ).
Table 1: General characteristics of patients enrolled in the study.

\begin{tabular}{|c|c|}
\hline No. of patients enrolled in the study & 154 \\
\hline Age: Mean \pm SD & 56.7 SD14.8 \\
\hline \multicolumn{2}{|l|}{ Gender: N. (\%) } \\
\hline - Men & $85(55.2 \%)$ \\
\hline - Women & $69(44.8 \%)$ \\
\hline Past episodes of acute cholecystitis: N. (\%) & $80(51.9 \%)$ \\
\hline Past episodes of acute pancreatitis: N. (\%) & $17(11 \%)$ \\
\hline Previous ERCP: N. (\%) & $11(7.1 \%)$ \\
\hline \multicolumn{2}{|l|}{ Clips used: N. (\%) } \\
\hline - Titanium clips & $100(64,9 \%)$ \\
\hline - Hem o Lock clips & $54(35,1 \%)$ \\
\hline WBC count 24 hours AS ( $\left.\times 10^{9} / \mathrm{I}\right)$ & 9.5 SD3.7 \\
\hline Neutrophils 24 hours AS (\%) & 75.3 SD8.2 \\
\hline CRP 24 hours AS (mg/l) & $5.2 \mathrm{SD} 4.8$ \\
\hline PA 24 hours AS (U/I) & 124.9 SD54.2 \\
\hline gGT 24 hours AS (U/I) & 58.6 SD83.9 \\
\hline Total bilirubin 24 hours AS (mg/dl) & 0.9 SD0.5 \\
\hline Hospitalization time: Days \pm SD & 3.0 SD4.5 \\
\hline Comorbidity: N. (\%) & $46(29.9 \%)$ \\
\hline \multicolumn{2}{|l|}{ Complications: N. (\%) } \\
\hline-7 days & $9(5.8 \%)$ \\
\hline - 30 days & $15(9.7 \%)$ \\
\hline - 180 days & $22(14.3 \%)$ \\
\hline
\end{tabular}

SD: Standard Deviation; ERCP: Endoscopic Retrograde CholangioPancreatography; WBC: White Blood Cell; AS: After Surgery; CRP: C-Reactive Protein; PA: Phosphatase Alkaline; gGT: gamma Glutamyl Transferase

Table 2: Demographic data and outcomes of two groups.

\begin{tabular}{|l|c|c|c|}
\hline & $\begin{array}{c}\text { Titanium clips } \\
\text { group } \\
\text { (n=100) }\end{array}$ & $\begin{array}{c}\text { Hem-o-lock } \\
\text { clips group } \\
\text { (n=54) }\end{array}$ & p-value \\
\hline Gender (M:F) & $54: 46$ & $31: 23$ & 0.7358 \\
\hline Age (years \pm SD) & 54.0 SD13.7 & 61.7 SD15.5 & 0.0018 \\
\hline WBC 24 hours AS (x 10\%/l) & 9.7 SD3.8 & 9.3 SD3.3 & 0.5155 \\
\hline Neutrophils 24 hours AS (\%) & 76.1 SD7.9 & 73.8 SD8.6 & 0.0968 \\
\hline CRP 24 hours AS (mg/l) & 6.1 SD5.2 & 3.5 SD3.4 & 0.0012 \\
\hline PA 24 hours AS (U/I) & 124.1 SD49.0 & 126.4 SD62.5 & 0.8015 \\
\hline gGT 24 hours AS (U/I) & 46.7 SD44.9 & 80.6 SD124.8 & 0.0157 \\
\hline Total bilirubin 24 hours AS(mg/dl) & 0.9 SD0.5 & 0.9 SD0.6 & 0.99 \\
\hline Hospitalization time (days \pm SD) & 3.6 SD5.5 & 2.0 SD0.7 & 0.0352 \\
\hline Comorbidity: No. (\%) & $31(31 \%)$ & $15(27.8 \%)$ & 0.7158 \\
\hline Complications: No. (\%) & 9 (9\%) & 0 (0\%) & 0.0273 \\
\hline - 7 days & $13(13 \%)$ & 9 (16.7\%) & 0.6305 \\
\hline - 30 days & & 5 (9.3\%) & 0.99 \\
\hline - 180 days & & & \\
\hline
\end{tabular}


Table 3: Complications after laparoscopic cholecystectomy after 7,30 , and 180 days.

\begin{tabular}{|c|c|c|c|}
\hline Complications after 7-days & $\begin{array}{l}\text { Titanium clips } \\
\text { group }(n=100)\end{array}$ & $\begin{array}{l}\text { Hem-o-lock } \\
\text { clips group } \\
\quad(n=54)\end{array}$ & p-value \\
\hline - Biliary leak: N. (\%) & $5(5 \%)$ & $0(0 \%)$ & 0.1628 \\
\hline - Intra abdominal bleeding: N. (\%) & $2(2 \%)$ & $0(0 \%)$ & 0.5416 \\
\hline - Intra abdominal abscess: N. (\%) & $0(0 \%)$ & $0(0 \%)$ & 0.99 \\
\hline - Wound infection: N. (\%) & $2(2 \%)$ & $0(0 \%)$ & 0.5416 \\
\hline \multicolumn{4}{|l|}{ Complications after 30-days } \\
\hline - Chronic abdominal pain: N. (\%) & $3(3 \%)$ & $3(5.6 \%)$ & 0.4236 \\
\hline - Intra abdominal abscess: N. (\%) & $4(4 \%)$ & $1(1.9 \%)$ & 0.6577 \\
\hline - Common bile duct stones: N. (\%) & $3(3 \%)$ & $1(1.9 \%)$ & 0.99 \\
\hline \multicolumn{4}{|l|}{ Complications after 180 -days } \\
\hline - Chronic abdominal pain: N. (\%) & $4(4 \%)$ & $4(7.4 \%)$ & 0.4521 \\
\hline - Gastrointestinal symptoms: N. (\%) & $8(8 \%)$ & $4(7.4 \%)$ & 0.99 \\
\hline - Common bile duct stones: N. (\%) & $1(1 \%)$ & $1(1.9 \%)$ & 0.99 \\
\hline
\end{tabular}

\section{Discussion}

LC is a surgical procedure considered relatively safe, but associated with a $\sim 0.3-0.5 \%$ mortality rate $[10], \sim 0.2-1.5 \%$ risk of bile duct injury [11-13] and $\sim 6$ - 9\% risk of major complications, such as myocardial infarction, heart failure, acute stroke, renal failure, pulmonary embolism, lung failure and postoperative shock [10].

Numerous devices have been introduced over the years to reduce the number of complications and length of hospital stay. Introduced in 1999, the HC has been a useful alternative to TC, endoloops, and endoscopic staplers [8].

There are four sizes of HCs and appliers ( $M, M L, L$, and $X L)$, from $2 \mathrm{~mm}$ to $16 \mathrm{~mm}$, for ligation of structures during cholecistectomy. The use of clips of appropriate size is important for proper ligation; too large a clip may not remain on the tissue owing to the relatively larger gap between the jaws of the locked clip.

This non-absorbable polymer locking clip presents advantages over the traditional TC: the lock engagement feature and the presence of teeth in the jaws provide good security and potentially reduce the chance of dislodgment from the tissue; it has a narrow profile, excellent tactile feedback with closure, removability, and absence of artifacts with imaging techniques. Loading of the applier with the clip is easy and a flexible mechanism virtually prevents clips from falling out of the applier [8, 14]. Moreover, they guarantee a complete circumferential dissection of the vessel, a visualization of the curved tip of the clip around and beyond the vessel and no cross-clipping, compared with the application of TC, squeezing clip handles too hard is unnecessary and, during transection of vessels, only a partial division is performed initially to confirm hemostasis before complete transaction [15].

There are several reports about the safety and feasibility of HCs in minimally invasive surgery. These devices are frequently used by urologists for ligation of the ureter, renal artery, and renal vein with good results $[15,16]$. HCs are contraindicated only for use in ligating the renal artery during laparoscopic nephrectomies in living donor patients [15].
In their experimental study of various traditional occlusion devices, Joseph and colleagues [17] showed the greater safety and security of HCs compared to TCs and endoscopic stapling devices, since as the HCs tolerated a pressure of $800 \mathrm{mmHg}$ on the arterial segment with no evidence of leak. Their study substantiates the use of HCs in vascular control so they can be used in hypertensive patients with no concern about slippage.

Moreover, HCs are safe and feasible for ligation of the appendiceal base and mesentery during laparoscopy appendectomy, with a similar result using single Hem-o-lok on the appendiceal stump $[18,19]$.

Several authors [20-22] respectively have published reports in favor of HCs during minimally invasive prostatectomy, hysterectomy, and lung lobectomy.

In our study, we presented the results of the application of HCs in relation to TCs during LC for gallbladder disease in 154 patients.

The Food and Drug Administration (FDA) Center for Devices and Radiological Health maintains a compendium of reports of adverse events involving medical devices called MAnufacturer and User facility Device Experience (MAUDE). It includes reports of adverse events involving medical devices that occur after device approval [23]. For the clinician who is considering the use of a new medical device, the MAUDE database is useful in searching for complications that have not been reported in the medical literature [24]. Overall, relatively few incidents of Hemo-Lok failure are documented in the MAUDE database. It is important to keep in mind that these are voluntary reports to the FDA, and a significant number of events may never be reported.

In our study, we observed no cases of clip failure during cholecystectomy. Problems with the HCs typically were not noted during the operation since the clips were apparently placed without issue, but subsequently they did not maintain control of the vessel. Thus, the problem in these cases was of unexpected bleeding during the early postoperative period. Difficulties during LC were relatively minor and amenable to laparoscopic salvage owing to the smaller vessels compared with urological procedures [8].

A recent article [25] presented an interesting case of acute cholangitis caused by surgical HC migration into the common bile duct with stone formation 13 months after LC. Such migration and the resulting stone formation is a rare but well-recognized complication of cholecystectomy.

Another interesting report [26] describe the case of a 62 -year-old woman who underwent LC because of gallstone disease with the appearance of the Mirizzi syndrome induced by the migration of polymer laparoscopic clips in the common bile duct.

Another report [27] described a 56-year-old woman who underwent laparoscopic left hepatectomy and cholecystectomy who was admitted to their hospital 16 months later with nausea and vomiting due to migration of $\mathrm{HC}$ endoclips and stitches into the duodenum; it is an incredibly rare report of a poorly understood mechanism.

In literature, there are several reports of $\mathrm{HC}$ migration during radical prostatectomy into the rectum [28] and urinary bladder, with subsequent bladder stone formation [29,30]. 
The number of HCs typically used per patient to ligate cystic duct and cystic artery is four, while TCs used during cholecystectomy are generally five. The cost of six HCs is US\$32, therefore not much more expensive than a TC blister.

On analyzing the data of our study, surgeons mainly used TCS; although the mean age of the TC group was lower than that of the $\mathrm{HC}$ group, the complication rate in the TC group was higher than in the $\mathrm{HC}$ group both 7 days after cholecystectomy, and 30 days, with a $p$-value statistically significant only for complication rate 7 days after surgery. Instead, complication rate 6 months after surgery was higher in the HC group. Specifically, leakage from the cystic duct was the most frequent complication in the TC group (5 patients, 5\%) compared to the HC group with a $p$-value not statistically significant $(p=0.1628)$, mainly owing to the relatively small number of patients enrolled in the study and the number of LC performed using HCs.

In the $\mathrm{HC}$ group, most frequent complication 180 days after surgery was chronic abdominal pain and gastrointestinal symptoms (4 patients, $7.4 \%$ ) with a $p$-value not statistically significant ( $p$-value $=0.4521$ and $p$-value $=0.99$, respectively).

The overall complication rate 7 days after surgery and mostly the percentage of leakage from the cystic duct in this study is higher than that described in the literature [11-13] probably owing to the number of cholecystectomies performed by the residents, for which the use of HCs could be recommended. The low number of complications 7 days after surgery in the HC group is in accordance with what emerged in a review of 2012 [14].

Another interesting finding that emerged from our study was the shorter mean hospital stay of $\mathrm{HC}$ patients owing to the lower number of post-operative complications and also to a postoperative course characterized by a faster normalization of the indices of inflammation (especially CRP), considered by us for discharge.

Possible limitations of this study are related to its retrospective, non-randomized design, which carries a high risk of selection bias. The single-institutional nature of the study, and the relatively small sample size could also affect the results.

Other possible limitations of the study are the percentage of cholecystectomies performed by the residents and the number needed to treat / number needed to harm ratio lower than 3.4. Therefore, the level of evidence behind the results of the present study is not high.

\section{Conclusions}

Hem-o-lock clips are used especially in urological surgery, with laparoscopic nephrectomy the most common operation. Based on our retrospective study, Hem-o-lock clips are safe, effective, and reliable for use during laparoscopic cholecystectomy. This study also showed that the complication rate 7 days after surgery was lower in the Hem-o-lock group with a shorter mean length of hospital stay. Therefore, we recommend using these clips during laparoscopic cholecystectomy for gallbladder disease and we encourage to continue to use this technique to gain more experience in a larger series of patients.

Future studies with a larger cohort will be necessary for a more accurate comparison between titanium clips and Hem-olock clips.
Authors' contributions: GP: Study conception and design, literature search, acquisition, interpretation and analysis of data. FF: Literature search, acquisition, interpretation and analysis of data. PL: acquisition, interpretation and analysis of data. RDA: drafting and critically revising the article for important intellectual content. All authors have read and approved the final manuscript.

Funding: The authors have not declared a specific grant for this research from any funding agency in the public, commercial or not-for-profit sectors.

Conflict of interest: The authors declare that they have no conflict of interest.

Acknowledgements: The authors thank Dr. David C. Nilson, Ph.D., for revising the English.

\section{Compliance with ethical standards}

Research involving human participants and/or animals: The study has been performed in accordance with the ethical standards of the institutional and/or national research committee, and with the 1964 Helsinki declaration and its later amendments or comparable ethical standards.

Informed consent: We have obtained consent to publish from the participants to report individual patient data.

\section{References}

1. Aerts R, Penninckx F. The burden of gallstone disease in Europe. Aliment Pharmacol Ther. 2003; 18: 49-53.

2. Everhart JE, Ruhl CE. Burden of digestive diseases in the United States Part III: Liver, biliary tract, and pancreas. Gastroenterology 2009; 136: 1134-1144.

3. Minutolo V, Licciardello A, Arena M, et al. Laparoscopic cholecystectomy in the treatment of acute cholecystitis: comparison of outcomes and costs between early and delayed cholecystectomy. Eur Rev Med Pharmacol Sci. 2014; 18: 40-6.

4. Festi D, Reggiani ML, Attili AF, et al. Natural history of gallstone disease: expectant management or active treatment? Results from a population-based cohort study. J Gastroenterol Hepatol 2010; 25: 719-724.

5. Kimura Y, Takada T, Strasberg SM, et al. TG13 current terminology, etiology, and epidemiology of acute cholangitis and cholecystitis. J Hepatobiliary Pancreat Sic. 2013; 20: 8-23.

6. Festi D, Reggiani ML, Attili AF, et al. Natural history of gallstone disease: expectant management or active treatment? Results from a population-based cohort study. J Gastroenterol Hepatol. 2010; 25: 719-724.

7. Junco SS, Mohandas S, Almond LM. Post cholecystectomy syndrome (PCS). Int J Surg. 2010; 8: 15-7.

8. Meng MV. Reported failures of the polymer self-locking (HemO-Lok) clip: review of data from the Food and Drug Administration. J Endourol. 2006; 20: 1054-7.

9. Yokoe M, Hata J, Takada T, Strasberg SM, et al. Tokyo Guidelines 2018: diagnostic criteria and severity grading of acute cholecystitis (with videos). J Hepatobiliary Pancreat Sci. 2018; 25: 41-54.

10. Huntington CR, Cox TC, Blair LJ, et al. Nationwide variation in outcomes and cost of laparoscopic procedures. Surg. Endosc. 2016; 30: 934-946.

11. Lill S, Rantala A, Pekkala E, et al. Elective laparoscopic cholecystectomy without routine intraoperative cholangiography: a 
retrospective analysis of 1101 consecutive cases. Scand. J. Surg. 2010; 99: 197-200.

12. Hamad MA, Nada AA, Abdel-Atty MY, Kawashti AS. Major biliary complications in 2,714 cases of laparoscopic cholecystectomy without intraoperative cholangiography: a multicenter retrospective study. Surg. Endosc. 2011; 25: 3747-3751.

13. Tornqvist B, Stromberg C, Persson G, Nilsson M. Effect of intended intraoperative cholangiography and early detection of bile duct injury on survival after cholecystectomy: population based cohort study. BMJ. 2012; 345; e6457.

14. Aminian A, Khorgami Z. Hem-o-Lok Clip is Safe in Minimally Invasive General Surgery: A Single Center Experience and Review of Data From Food and Drug Administration. J Minim Invasive Surg Sci. 2012; 1: 52-7.

15. Ponsky L, Cherullo E, Moinzadeh A, et al. The Hem-o-lok clip is safe for laparoscopic nephrectomy: a multi-institutional review. Urology. 2008; 71: 593-6.

16. Kapoor R, Singh KJ, Suri A, et al. Hem-o-lok clips for vascular control during laparoscopic ablative nephrectomy: a single-center experience. J Endourol. 20: 202-204.

17. Joseph J, Leung Y-Y, Eichel L, Scheidweiler K, Erturk E, Wood R. Comparison of the Ti-knot device and Hem-o-lok clips with other devices commonly used for laparoscopic renal artery ligation. J Endourol. 2004; 18: c163-166.

18. Koluh A, Delibegovic S, Hasukic S, Valjan V, Latic F. Laparoscopic appendectomy in the treatment of acute appendicitis. Med Arh. 2010; 64: 147-50.

19. Partecke LI, Kessler W, von Bernstorff W, Diedrich S, Heidecke $\mathrm{CD}$, Patrzyk M. Laparoscopic appendectomy using a single polymeric clip to close the appendicular stump. Langenbecks Arch Surg. 2010; 395: 1077-82.

20. Yi JS, Kwak C, Kim HH, Ku JH. Surgical clip-related complications after radical prostatectomy. Korean J Urol. 2010; 51: 683-7.

21. Feuer $G$, Hernandez P, Barker J. Surgical technique enhances the efficiency of robotic hysterectomy. Int J Med Robot. 2011; 7: 1-6.
22. Bignon $\mathrm{H}$, Buela E, Martinez-Ferro M. Which is the best vesselsealing method for pediatric thoracoscopic lobectomy? J Laparoendosc Adv Surg Tech A. 2010; 20: 395-8.

23. U. S. Food and Drug Administration. MAUDE - Manufacturer and User Facility Device Experience. 2011.

24. Gurtcheff SE. Introduction to the MAUDE database. Clin Obstet Gynecol. 2008; 51: 120-3.

25. Roh YJ, Kim JW, Jeon TJ, Park JY. Common bile duct stone development due to a Hem-o-lok clip migration: A rare complication of laparoscopic cholecystectomy. BMJ Case Rep. 2019; 12.

26. Nagorni EA, Kouklakis G, Tsaroucha A, et al. Post-laparoscopic cholecystectomy Mirizzi syndrome induced by polymeric surgical clips: a case report and review of the literature. J Med Case Rep. 2016; 10: 135.

27. Xia Y, Gao XF, Shi CY, Jiang YH, Yi X. Migration of hem-o-lock clips and stitches into the duodenum after laparoscopic hepatectomy and cholecystectomy. Medicine (Baltimore). 2019; 98: e18153.

28. Wu SD, Rios RR, Meeks JJ, Nadler RB. Rectal Hem-o-Lok clip migration after robot-assisted laparoscopic radical prostatectomy. Can J Urol. 2009; 16: 4939-40.

29. Moser RL, Narepalem N. Erosion of Hem-o-Lok clips at the bladder neck after robot-assisted radical prostatectomy. J Endourol. 2009; 23: 949-51.

30. Mora ER, Gali OB, Garin JA, Arango O. Intravesical migration and spontaneous expulsion of a Hem-o-lok polymer ligating clip after laparoscopic radical prostatectomy. Urology. 2010; 75: 1317. 